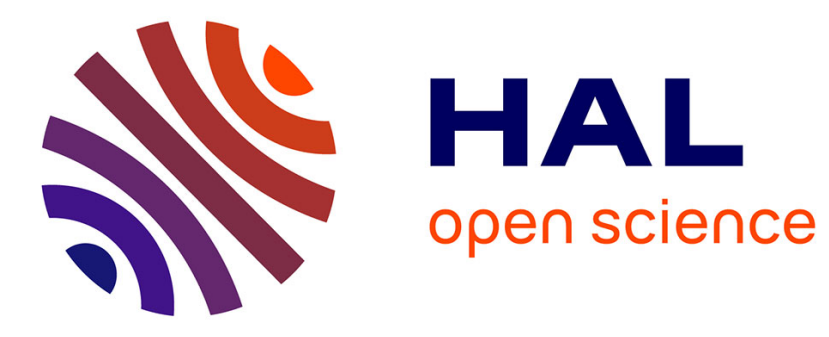

\title{
Mutational analysis of the Qi-site proton pathway in yeast cytochrome bc1 complex
}

Zehua Song, Yangfeng Hu, Bogdan I. Iorga, Cindy Vallières, Nicholas Fisher, Brigitte Meunier

\section{- To cite this version:}

Zehua Song, Yangfeng Hu, Bogdan I. Iorga, Cindy Vallières, Nicholas Fisher, et al.. Mutational analysis of the Qi-site proton pathway in yeast cytochrome bc1 complex. Biochemical and Biophysical Research Communications, 2020, 523 (3), pp.615-619. 10.1016/j.bbrc.2019.12.102 . hal-02445554

\section{HAL Id: hal-02445554 \\ https://hal.science/hal-02445554}

Submitted on 10 Nov 2020

HAL is a multi-disciplinary open access archive for the deposit and dissemination of scientific research documents, whether they are published or not. The documents may come from teaching and research institutions in France or abroad, or from public or private research centers.
L'archive ouverte pluridisciplinaire HAL, est destinée au dépôt et à la diffusion de documents scientifiques de niveau recherche, publiés ou non, émanant des établissements d'enseignement et de recherche français ou étrangers, des laboratoires publics ou privés. 
Mutational analysis of the $\mathrm{Q}_{\mathrm{i}}$-site proton pathway in yeast cytochrome $b c_{1}$ complex

Zehua Song ${ }^{\mathrm{a}}$, Yangfeng $\mathrm{Hu}^{\mathrm{a}}$, Bogdan I. Iorga ${ }^{\mathrm{b}}$ Cindy Vallières $^{\mathrm{c}}$, Nicholas Fisher ${ }^{\mathrm{d}}$, and Brigitte Meunier ${ }^{\mathrm{e}}$

Affiliation:

a Translational Research Institute, Henan Provincial People's Hospital, School of Medicine, Henan University, Zhengzhou, China

b Université Paris-Saclay, CNRS, Institut de Chimie des Substances Naturelles, UPR 2301, 91198, Gif-sur-Yvette, France.

c School of Life Sciences, University of Nottingham, University Park, Nottingham, NG7 2RD, UK.

d MSU-DOE Plant Research Laboratory, Michigan State University, East Lansing, MI 48824, USA.

e Institute for Integrative Biology of the Cell (I2BC), CEA, CNRS, Université Paris-Saclay, 91198, Gif-sur-Yvette, France

\section{Corresponding authors:}

Brigitte Meunier Institute for Integrative Biology of the Cell (I2BC), 1 avenue de la Terrasse, 91198 Gif-sur-Yvette, France

brigitte.meunier@i2bc.paris-saclay.fr

Nicholas Fisher, MSU-DOE Plant Research Lab, 612 Wilson Road, East Lansing, MI 48824

nefisher@msu.edu 


\section{Summary}

The respiratory cytochrome $b c_{1}$ complex functions as a protonmotive ubiquinol:cytochrome $c$ oxidoreductase. Lysine 228 (K228) located within the quinol reduction $\left(\mathrm{Q}_{\mathrm{i}}\right)$ site of the $b c_{1}$ complex, has been reported as a key residue for proton transfer during the redox chemistry cycle to substrate quinone at $\mathrm{Q}_{\mathrm{i}}$. In yeast, while single mutations had no effect, the combination of $\mathrm{K} 228 \mathrm{~L}$ and $\mathrm{F} 225 \mathrm{~L}$ resulted in a severe respiratory growth defect and inhibition of $\mathrm{O}_{2}$ consumption in intact cells. The inhibition was overcome by uncoupling the mitochondrial membrane or by suppressor mutations in the region of K228L-F225L. We propose that the K228L mutation introduces energetic (and kinetic) barriers into normal electron- and proton transfer chemistry at $\mathrm{Q}_{\mathrm{i}}$, which are relieved by dissipation of the opposing protonmotive force or through the restoration of favourable intraprotein proton transfer networks via suppressor mutation.

Keywords: proton/electron transfer, respiratory complex, complex III catalytic activity, mitochondrial mutations.

\section{Introduction}

The cytochrome $b c_{1}$ complex of the mitochondrial respiratory chain catalyses the oxidation of ubiquinol and the reduction of cytochrome $c$, which is coupled to the movement of proton across the mitochondrial inner membrane.

Three subunits form the catalytic core of this multimeric enzyme: cytochrome $c_{1}$, the ironsulfur protein and cytochrome $b$. The latest, mitochondrially encoded in all eukaryotes, is the central and largest subunit, consisting of eight transmembrane helices and providing the two ubiquinol (and inhibitor) binding sites, the quinol oxidation site $\left(\mathrm{Q}_{\mathrm{o}}\right)$ and the quinone reduction site $\left(\mathrm{Q}_{\mathrm{i}}\right)$.

These two sites are located on opposite sides of the membrane and linked by a transmembrane electron-transfer pathway (via hemes $b_{l}$ and $b_{h}$ ). An ubiquinol molecule binds at the $\mathrm{Q}_{\mathrm{o}}$-site, is deprotonated, and transfers one electron through the iron-sulphur protein and cytochrome $c_{1}$ to cytochrome $c$, forming a semiquinone. The semiquinone then reduces $b_{l}$ which in turn functions as reductant for $b_{h}$. Ferroheme $b_{h}$ then reduces the ubiquinone bound at the $\mathrm{Q}_{\mathrm{i}}$-site, forming a stable (tightly bound) semiquinone species, with associated proton uptake from the mitochondrial matrix. A second ubiquinol oxidation at the $\mathrm{Q}_{0}$-site completes the cycle with the formation of fully reduced ubiquinol at the $\mathrm{Q}_{\mathrm{i}}$-site. The oxidation and reduction of ubiquinol/ubiquinone require proton movement to and from the aqueous phase, and thus proton pathways that are not as well defined as electron transfer paths.

Residue K228 (yeast (Saccharomyces cerevisae) numbering) is a key residue of a pathway for the uptake of protons from the matrix into the Qi-site, first termed cardiolipin /K-pathway [1]. In this pathway, K228 would acquire a proton from the bound cardiolipin, then passes it via water molecules into the $\mathrm{Q}_{\mathrm{i}}$-site for the reduction of quinone. Molecular dynamics studies suggests that the sidechain of $\mathrm{K} 228$, once protonated, rotates into the $\mathrm{Q}_{\mathrm{i}}$-site to form a saltbridge with D229 [2]. In the bacterial enzyme, replacement of both K228 and D229 by non- 
protonable residues fully blocked the $\mathrm{Q}_{\mathrm{i}}$-site function by disabling the proton transfer path [3].

K228 is highly conserved amongst species. However in yeast, the replacement of K228 by a methionine, isoleucine or leucine is well tolerated and the enzyme is active (see for instance [4] and references within). Nearby protonatable residues H202 and /or D229 and/or water molecules might take over K228 activity and render the residue dispensable. Interestingly in the human malaria parasite Plasmodium falciparum and other apicomplexans, a leucine replaces K228. In addition, while H202 and D229 are conserved, other amino-acid substitutions (as compared to yeast) are found in the vicinity.

In our attempts to reconstruct the $\mathrm{Q}_{\mathrm{i}}$-site of yeast $b c_{1}$ complex to render the pocket more similar to the P.falciparum $\mathrm{Q}_{\mathrm{i}}$-site (and thus a potentially convenient model for the parasite enzyme), we found that residues cannot be interchanged without deleterious effect. Here, we studied the effect of Plasmodium-like substitutions of K228 and neighbour residues. The combination of $\mathrm{K} 228 \mathrm{~L}$ and $\mathrm{F} 225 \mathrm{~L}$, naturally present in P.falciparum, caused a severe respiratory growth defect while the $b c_{1}$ complex remained apparently functional in uncoupled mitochondria. The growth defect can be overcome by suppressor mutations in the vicinity of the primary mutations.

\section{Materials and Methods}

\section{Materials and growth media}

Equine cytochrome $c$, decylubiquinone, superoxide dismutase and catalase were obtained from Sigma Aldrich. The following media were used for the growth of yeast: YPD (1\% yeast extract, $2 \%$ peptone and $3 \%$ glucose), YPGal (1\% yeast extract, $2 \%$ peptone and $2 \%$ galactose) and YPG (1\% yeast extract, $2 \%$ peptone and $2 \%$ glycerol).

\section{Yeast mutants}

The multiple mutations were introduced in yeast cytochrome $b$ by side-directed mutagenesis and mitochondrial transformation [5,6]. In all experiments, control and mutants have identical nuclear and mitochondrial genomes with the exception of the mutations introduced in the cytochrome $b$ gene.

\section{Measurement of decylubiquinol-cytochrome $c$ reductase activity}

Yeast mitochondria were prepared as in [7]. Concentration of $b c_{1}$ complex in the mitochondrial samples was determined from dithionite-reduced optical spectra, using $\varepsilon=28.5$ $\mathrm{mM}^{-1} \mathrm{~cm}^{-1}$ at $562 \mathrm{~nm}$ minus $575 \mathrm{~nm}$.

Decylubiquinol-cytochrome $c$ reductase activities were determined at room temperature by measuring the reduction of cytochrome $c$ (final concentration of $20 \mu \mathrm{M}$ ) at $550 \mathrm{~nm}$ versus 540 $\mathrm{nm}$ over one-min time-course in $10 \mathrm{mM}$ potassium phosphate $\mathrm{pH} \mathrm{7,0.01 \%} \mathrm{(w/v)} \mathrm{lauryl-}$ maltoside and $2 \mathrm{mM} \mathrm{KCN}$. Mitochondria were added to obtain a final concentration of 5-15 $\mathrm{nM} b c_{1}$ complex. Activity was initiated by the addition of decylubiquinol. Each measurement was repeated three to five times and the values obtained were averaged. Activities $\left(k_{c a t}\right)$ were determined as the cytochrome $c$ reduction rate per $b c_{1}$ complex.

Apparent $\mathrm{K}_{\mathrm{M}}$ values were estimated from the plots of cytochrome $c$ reduction rates $v s$ decylubiquinol concentrations, as the decylubiquinol concentrations required to obtain $50 \%$ of 
the observed maximum rate of cytochrome $c$ reduction. In order to assess the production of superoxide (SO), cytochrome $c$ reduction rates were recorded in absence and in presence of superoxide dismutase and catalase, both at $225 \mathrm{units} / \mathrm{mL}$, in potassium phosphate buffer 50 $\mathrm{mM}$ pH 7 with $0.1 \mathrm{mM} \mathrm{KCN}$. The measurements were repeated at least three times and the obtained values averaged.

\section{Aconitase and fumarase measurement using cell extracts}

The aconitase and fumarase activities were determined spectrophotometrically by monitoring the formation of cis-aconitate and fumarate at $240 \mathrm{~nm}$ and $25^{\circ} \mathrm{C}$. Briefly, cell extracts were prepared from $2.0 \times 10^{8}$ cells $\left(\mathrm{OD}_{600} \sim 20\right)$ grown on YPGal. Lysis was performed at $4^{\circ} \mathrm{C}$ in 10 $\mathrm{mM}$ MES buffer, pH6 containing $0.6 \mathrm{mM} \mathrm{MnCl}_{2}$ and deprived of oxygen (by bubbling with nitrogen gas) with $0.5 \mathrm{~mm}$ glass beads (v/v), by vortexing for $30 \mathrm{~s}$ followed by incubation on ice for $30 \mathrm{~s}$, repeating the process seven times. Cell debris was removed by centrifugation at $13,000 \mathrm{rpm}$ for $5 \mathrm{~min}$, and the resulting supernatant was aliquoted and frozen immediately in liquid nitrogen and kept at $-80^{\circ} \mathrm{C}$. Samples were thawed just before the assay. Protein concentration was determined spectrophotometrically with the Bio-Rad protein assay kit, according to the manufacturer's instructions.

For the aconitase activity, the assay mixture contained $50 \mathrm{mM}$ potassium phosphate buffer, $\mathrm{pH} 7.4,30 \mathrm{mM}$ sodium isocitrate, $0.6 \mathrm{mM} \mathrm{MnCl}_{2}$, and $150-250 \mu \mathrm{g}$ of protein for a final volume of $1 \mathrm{ml}$. For the fumarase activity, the assay mixture contained $50 \mathrm{mM}$ potassium phosphate buffer, $\mathrm{pH} \mathrm{7.4,50} \mathrm{mM} \mathrm{L-malic} \mathrm{acid} \mathrm{and} \mathrm{150-250} \mu \mathrm{g}$ of protein for a final volume of $1 \mathrm{ml}$. The absorbance changes were measured for $20 \mathrm{~min}$, and the activity was calculated from the slope of the linear portion; $\varepsilon_{240}=3.6 \mathrm{mM}^{-1} \mathrm{~cm}^{-1}$ for cis-aconitate and $\varepsilon_{240}=2.44$ $\mathrm{mM}^{-1} \mathrm{~cm}^{-1}$ for fumarate.

\section{Measurement of oxygen consumption by intact cells}

Yeast were cultivated in YPGal medium at $28^{\circ} \mathrm{C}$ in flasks with vigorous agitation for a good aeration. Cells were harvested at an $\mathrm{OD}_{600} \mathrm{~nm}$ of 15-18. After centrifugation, the cells were resuspended in fresh YPGal to an $\mathrm{OD}_{600} \mathrm{~nm}$ of around 250 and immediately used for the assay. Oxygen consumption was monitored in YPGal using an oxygen electrode at $25^{\circ} \mathrm{C}$ before and after the addition of $15 \mu \mathrm{M}$ CCCP (carbonyl cyanide m-chlorophenyl hydrazine). The measurements were repeated at least three times and the values averaged. The oxygen consumption activities were determined as oxygen uptake rates per $\mathrm{OD}_{600 \mathrm{~nm}}$ cells.

\section{Results}

\section{Plasmodium-like mutations in proton pathway of yeast $b c_{1}$ complex $\mathrm{Q}_{\mathrm{i}}$-site}

In the region of the proton-pathway leading to the Qi-site, two residues that differ between yeast and P.falciparum seemed particularly interesting to study, namely residues 228 and 225 . These residues are located at the entry towards the Qi-site. Analysis of the large collection of eukaryote cytochrome $b$ sequence publicly available (over 8,500) showed that these two residues are conserved. K228 is present in $98.8 \%$ of the analysed sequences but it is replaced by leucine in P.falciparum. Position 225 is occupied by an aromatic residue in most of the organisms (phenylalanine in $68.5 \%$, tyrosine in $27.9 \%$ or histidine in $1.7 \%$ ) whereas it is also a leucine in the parasite. D229 that would connect with K228 for proton transfer [3], and 
H202 and S206 possibly involved in substrate binding and protonation are conserved between yeast and P.falciparum.

Single mutants F225L and K228L were already available and previously studied. We produced the double mutant F225L - K228L, and a mutant, named PFQi11 that combined F225L - K228L with two other changes, namely M221F and V233F. Residue 233, (valine in yeast and phenylalanine in the parasite) is not well conserved and is not sensu stricto in the $\mathrm{Q}_{\mathrm{i}}$-site although its sidechain points towards bound antimycin. Residue 221 is a methionine in yeast while it is a phenylalanine in P. falciparum and in most of the organisms (97.2\%). The position of the residues are shown in Fig.1

\section{Respiratory growth competence and $b c_{1}$ complex activity}

We had previously tested the effect of single mutations M221F, F225L and K228L [8]. The mutations had no or minor effect on growth competence and $b c_{1}$ complex activity. The effect of $\mathrm{V} 233 \mathrm{~F}$ alone was not tested. The mutation was unlikely to have a deleterious effect, the residue being located slightly outside the $\mathrm{Q}_{\mathrm{i}}$-site.

By contrast, the combination F225L and K228L caused a severe growth defect. PFQi11 that harbours F225L and K228L with M221 and V233F was also unable to grow on respiratory medium (Fig.2).

Mitochondria were then prepared from the two mutants and the $b c_{1}$ complex activity was monitored. Surprisingly, PFQi11 showed a WT $b c_{1}$ complex activity and the double mutant presented only a two-fold decreased activity which could not account for the complete respiratory growth defect (Table 1).

$\mathrm{K}_{\mathrm{M}}$ for decylubiquinol were estimated as described in Materials and Methods. PFQi11, the double mutant F225L- K228L, and the single mutant K228L showed a slightly higher $\mathrm{K}_{\mathrm{M}}$ of 7-8 $\mu \mathrm{M}$. $\mathrm{K}_{\mathrm{M}}$ for F225L was as WT. The slight difference in $\mathrm{K}_{\mathrm{M}}$ values did not suggest severe alterations in the binding environment or the accessibility of the quinol/quinone into the site.

We then checked whether the apparent high rate of cytochrome $c$ reduction observed in PFQi11 and the double mutant F225L- K228L might be due to SO over-production by the $b c_{1}$ complex during the assay. We thus monitored SO production as the superoxide dismutasesensitive rate of cytochrome $c$ reduction (Materials and Methods). The difference between the reduction rate in the absence and the presence of added superoxide dismutase gives the contribution of the cytochrome $c$ reduction by SO to the overall cytochrome $c$ reductase activity. No SO production was detected (not shown), in contrast to the observed SO overproduction caused by Plasmodium-like mutations in the $\mathrm{Q}_{0}$-site [9] Thus there was no indication of a severe Q-cycle dysfunction in the PFQi11 and F225L- K228L $b c_{1}$ complex, in the conditions of the assays, explaining the respiratory growth defect.

\section{Suppressor mutations}

We then investigated ways to overcome the growth defect. Starting with PFQi11, we selected colonies with restored respiratory growth. To that end, several subclones of the mutant were incubated on YPG medium. After two to four weeks, respiratory competent colonies appeared. Eight independent colonies (each from different subclones) were analysed. The 
suppressor mutations, identified by sequencing the cytochrome $b$ gene were T232I (found in four colonies); N31D, Y28S, K228M and the reversion back to WT of residue F225 (observed once). The location of the primary mutations and suppressors is shown in Fig.1.

The residue at position 28 is not strongly conserved amongst organisms, although it is mostly a tyrosine or a phenylalanine in fungi and protists, but a serine is found in $15 \%$ of animal cytochrome $b$. N31 is well conserved as it is found in $94.7 \%$ of the analysed sequences. An aspartate was not reported at that position. At position 232, a glycine is found in $90.1 \%$ of the sequences but it is a threonine in most of the fungi. In protists, the position is occupied by glycine, alanine or serine. An isoleucine was not reported. Thus the secondary mutations introduced original residues in that region of the Qi-site that resulted in a restored function.

The respiratory growth competence and $b c_{1}$ complex activities of the suppressors were assessed (Fig.2 and Table 1). The secondary mutations restored a good growth. The $b c_{1}$ complex activities varied from a two-fold decreased activity as compared to WT (27 $\mathrm{s}^{-1}$ SUP2) to WT level (SUP1 and SUP6). The $\mathrm{K}_{\mathrm{M}}$ for quinol was decreased (3 to $5 \mu \mathrm{M}$ ) and thus closer or identical to WT. None of the suppressors showed SO overproduction in the $b c_{1}$ complex assay (not shown).

\section{Oxygen consumption of cells}

In order to obtain more information on the cause of the growth defect in PFQi11 and F225LK228L, we monitored the oxygen consumption activity of freshly grown WT and mutant cells.

PFQi11 and F225L-K228L displayed low oxygen consumption activities under physiological conditions (without added uncoupler): $30 \%$ and $7 \%$ of the WT rate, respectively (Fig.3A), which clearly reflects the respiratory growth deficiency. Upon CCCP addition (Fig.3B), a tenfold increase in oxygen consumption rate was observed in the mutant cells, compared to the three-fold increase observed with WT cells. Thus in presence of CCCP, PFQi11 oxygen consumption reached WT level. F225L-K228L rate remained lower (28\% of WT rate). The single mutation F225L and K228L had no or much milder effect and their behaviour was closer to that of the WT.

The oxygen consumption activities of the suppressors were also tested. As showed in Fig. 3, the suppressor mutations restored normal oxygen consumption activity and reactivity to CCCP.

These data suggested that in PFQi11, the $b c_{1}$ complex activity, and by consequent the respiratory chain, were blocked under normal physiological conditions, i.e. in untreated cells with coupled mitochondria. Addition of CCCP released that inhibition as the $\mathrm{O}_{2}$ consumption activity in CCCP treated cells reached WT rate.

It should be noted that PFQi11 $b c_{1}$ complex activities found to reach WT level (Table 1) were assayed in buffer without osmotic protection and with detergent, thus on broken uncoupled mitochondrial membranes.

The double mutant F225L-K228L displayed the same behaviour as PFQi11 but with a more severe defect. Thus the two additional changes, M221F and V233F, seem to attenuate the deleterious effect of F225L-K228L. 


\section{Effect of PFQi11 mutations on ROS production in cells}

In the mutant cells (with intact mitochondria), the $\mathrm{Q}_{\mathrm{i}}$-site mutations could slow the reduction of quinone, resulting in electron accumulation on heme $b_{1}$. This could subsequently result in ROS generation from $\mathrm{Q}_{0}$-bound semiquinone, similarly to the antimycin-inhibited state (see for instance [10] and references within). We thus investigated whether ROS were overproduced in PFQi11 and F225L- K228L cells as a result of inhibited $b c_{1}$ complex.

We measured the activity of aconitase, a tricarboxylic acid (TCA) cycle, located in the mitochondrial matrix and known to be highly sensitive to ROS damage. As a control, the activity of the TCA enzyme fumarate that is not sensitive to oxidative attack was monitored in parallel. As shown in Fig.4, the aconitase activity was over two-fold decreased in the mutants, clearly indicating an increased ROS level.

As mentioned above, SO production was not observed in the $b c_{1}$ complex assays, which was performed on broken uncoupled mitochondria.

The data argue for a dysfunction of the $b c_{1}$ complex in cells.

The increased oxidative stress in the mutants could also be due to ROS production by the NADH- and succinate-dehydrogenases. The $b c_{1}$ complex activity being inhibited in the mutant cells, electrons are expected to accumulate on the redox centres of upstream respiratory enzymes, with possible subsequent leak to oxygen.

\section{Discussion}

The combination of the $\mathrm{Q}_{\mathrm{i}}$-site mutations $\mathrm{K} 228 \mathrm{~L}$ and $\mathrm{F} 225 \mathrm{~L}$ resulted in a blocked $\mathrm{O}_{2}$ consumption in intact cells (i.e. with intact coupled mitochondria). The inhibition was released 1) upon addition of the uncoupler CCCP that collapsed the transmembrane protonmotive force and in broken uncoupled mitochondria; 2) by suppressor mutations in the vicinity of F255L-K228L.

We hypothesize that proton entry into the $\mathrm{Q}_{\mathrm{i}}$-site would be hindered by the double mutation, which would slow down the electron transfer- and quinone reduction chemistry at the $\mathrm{Q}_{\mathrm{i}}$-site. This is not the limiting step of the overall reaction catalysed by the $b c_{1}$ complex (which is the first electron transfer reaction at the $\mathrm{Q}_{0}$-site [11]). This offers an explanation for the WT rate of cytochrome $c$ reduction observed in the assays with broken mitochondria prepared from PFQi11 (Table 1). In intact cells with coupled mitochondria however, the thermodynamic backpressure of the membrane potential would be expected to further retard proton entry and (electrogenic) electron flow to the $\mathrm{Q}_{\mathrm{i}}$-site, which would result in a severe decrease in $b c_{1}$ complex activity and thus in respiratory function. The thermodynamic backpressure affecting transmembrane electron transfer to $b_{\mathrm{h}}$ (or proton uptake at the $\mathrm{Q}_{\mathrm{i}}$-site [12]) would be released on addition of an uncoupler (such as CCCP) or in broken mitochondrial membrane preparations.

Delay in protonation of the semiquinone anion would make the second electron transfer from heme $b_{\mathrm{h}}$ energetically unfavourable [13]. This would be expected to result in ROS production due to the accumulation of electrons on the low potential chain within 
cytochrome $b$ and increased occupancy of semiquinone in the $\mathrm{Q}_{\mathrm{o}}$-site, as observed in the mutant cells (Fig.4). The redox potential of the quinone/semiquinone couple in the $\mathrm{Q}_{\mathrm{i}}$-site might be changed due to minor structural perturbation of the substrate binding site, altering the equilibrium constant for electron transfer from ferroheme $b_{\mathrm{h}}$ and favouring electron accumulation in the low potential chain within cytochrome $b$. Similarly, the redox potential of heme $b_{\mathrm{h}}$ could also be altered by the mutations although residues $\mathrm{F} 225 / \mathrm{K} 228$ are quite far from heme $b_{\mathrm{h}}(8 \AA$ closest approach), as has been observed in mutants of the Rhodobacter sphaeroides $b c_{1}$ complex [14]. We note that the redox potential of $b_{\mathrm{h}}$ is in itself likely to vary during the reaction cycle in the $\mathrm{Q}{ }$-site by around $+100 \mathrm{mV}$ on the basis of semiquinone occupancy and proximity, and that this phenomenon may be affected by mutations altering the local fold and protein environment at $\mathrm{Q}_{i}[12,14,15]$.

It may also be suggested that the F255L-K228L mutations change the distribution of $\mathrm{Q}_{\mathrm{i}}$-bound water (and/or the $\mathrm{pKa}$ values of nearby proton carriers, which may include phospholipid), introducing a kinetic barrier for reduction and protonation of semiquinone [1619]. In the suppressors, respiratory function is restored by either replacing the leucine at position 225 by the original phenylalanine, by replacing the leucine at position 228 by a methionine or by introducing changes in other residues, namely Y28, N31 or T232. The amino-acid substitutions may restore the proper water molecule (or general proton transfer) network for efficient redox chemistry at $\mathrm{Q}_{\mathrm{i}}$. However a solely kinetic problem is unlikely to explain the rescue of the $b c_{1}$ complex activity by CCCP and broken (uncoupled) mitochondrial membrane preparations. Thermodynamic factors arising from unfavourable heme (or quinone/semiquinone) redox potentials due to the introduced residues are also likely to play an important role in the diagnosis of these mutants.

\section{Acknowledgements}

This work was in part funded by the CNRS and Chemical Sciences, Geosciences and Biosciences Division, Office of Basic Energy Sciences, Office of Science, U.S. Department of Energy (award number DE-FG02-91ER20021).

\section{References}

[1] C. Hunte, H. Palsdottir, B.L. Trumpower, Protonmotive pathways and mechanisms in the cytochrome $b c_{1}$ complex, FEBS Lett. 545 (2003) 39-46. doi:10.1016/S00145793(03)00391-0.

[2] P.A. Postila, K. Kaszuba, P. Kuleta, I. Vattulainen, M. Sarewicz, A. Osyczka, T. Róg, Atomistic determinants of co-enzyme $\mathrm{Q}$ reduction at the $\mathrm{Q}_{\mathrm{i}}$-site of the cytochrome $b c_{1}$ complex, Sci. Rep. 6 (2016) 1-10. doi:10.1038/srep33607.

[3] P. Kuleta, M. Sarewicz, P. Postila, T. Róg, A. Osyczka, Identifying involvement of Lys251/Asp252 pair in electron transfer and associated proton transfer at the quinone reduction site of Rhodobacter capsulatus cytochrome $b c_{1}$, Biochim. Biophys. Acta Bioenerg. 1857 (2016) 1661-1668. doi:10.1016/j.bbabio.2016.07.003.

[4] P. Mounkoro, T. Michel, R. Benhachemi, G. Surpateanu, B.I. Iorga, N. Fisher, B. 
Meunier, Mitochondrial complex III $\mathrm{Q}_{\mathrm{i}}$-site inhibitor resistance mutations found in laboratory selected mutants and field isolates, Pest Manag. Sci. 75 (2019) 2107-2114. doi:10.1002/ps.5264.

[5] P. Hill, J. Kessl, N. Fisher, S. Meshnick, B.L. Trumpower, B. Meunier, Recapitulation in Saccharomyces cerevisiae of cytochrome $b$ mutations conferring resistance to atovaquone in Pneumocystis jiroveci, Antimicrob. Agents Chemother. 47 (2003) 27252731. doi:10.1128/AAC.47.9.2725.

[6] N. Fisher, C.K. Castleden, I. Bourges, G. Brasseur, G. Dujardin, B. Meunier, Human disease-related mutations in cytochrome $b$ studied in yeast, J. Biol. Chem. 279 (2004) 12951-8. doi:10.1074/jbc.M313866200.

[7] C. Lemaire, G. Dujardin, Preparation of respiratory chain complexes from Saccharomyces cerevisiae wild-type and mutant mitochondria: activity measurement and subunit composition analysis, Methods Mol. Biol. 432 (2008) 65-81. doi: 10.1007/978-1-59745-028-7_5.

[8] Z. Song, B.I. Iorga, P. Mounkoro, N. Fisher, B. Meunier, The antimalarial compound ELQ-400 is an unusual inhibitor of the $b c_{1}$ complex, targeting both $\mathrm{Q}_{0}$ and $\mathrm{Q}_{\mathrm{i}}$ sites, FEBS Lett. 592 (2018)1346-1356. doi:10.1002/1873-3468.13035.

[9] Z. Song, J. Clain, B.I. Iorga, C. Vallières, A. Lalève, N. Fisher, B. Meunier, Interplay between the hinge region of Iron Sulfur Protein and the $\mathrm{Q}_{\mathrm{o}}$ site in the $b c_{1}$ complex Analysis of Plasmodium-like mutations in the yeast enzyme, Biochim. Biophys. Acta Bioenerg. 1847 (2015) 1487-1494. doi:10.1016/j.bbabio.2015.08.005.

[10] L. Bleier, S. Dröse, Superoxide generation by complex III: from mechanistic rationales to functional consequences, Biochim. Biophys. Acta. 1827 (2013) 1320-31. doi:10.1016/j.bbabio.2012.12.002.

[11] A.R. Crofts, B. Barquera, R.B. Gennis, R. Kuras, M. Guergova-kuras, E.A. Berry, Mechanism of ubiquinol oxidation by the $b c_{1}$ complex : Different domains of the quinol binding pocket and their role in the mechanism and binding of inhibitors, Biochemistry 38 (1999) 15807-15826. doi:10.1021/bi990962m

[12] P.R. Rich, A.E. Jeal, S.A. Madgwick, A.J. Moody, Inhibitor effects on redox-linked protonations of the $b$ haems of the mitochondrial $b c_{1}$ complex, Biochim. Biophys. Acta - Bioenerg. 1018 (1990) 29-40. doi:10.1016/0005-2728(90)90106-E.

[13] P.R. Rich, Electron and proton transfers through quinones and cytochrome $b c$ complexes, Biochim. Biophys. Acta-Bioenerg. 768 (1984) 53-79. doi:10.1016/03044173(84)90007-7.

[14] B. Hacker, B. Barquera, A.R. Crofts, R.B. Gennis, Characterization of mutations in the cytochrome $b$ subunit of the $b c_{1}$ complex of Rhodobacter sphaeroides that affect the quinone reductase site $\left(\mathrm{Q}_{\mathrm{c}}\right)$, Biochemistry 32 (1993) 4403-4410. doi:10.1021/bi00067a033.

[15] A.R. Crofts, The cytochrome $b c_{1}$ complex: Function in the context of structure, Annu. Rev. Physiol. 66 (2004) 689-733. doi:10.1146/annurev.physiol.66.032102.150251.

[16] E. A. Berry, L.S. Huang, Z. Zhang, S.H. Kim, Structure of the avian mitochondrial cytochrome $b c_{1}$ complex, J. Bioenerg. Biomembr. 31 (1999) 177-90.

doi:10.1023/a:1005459426843 
[17] X. Gao, X. Wen, L. Esser, B. Quinn, L. Yu, C.-A. Yu, D. Xia, Structural basis for the quinone reduction in the $b c_{1}$ complex: a comparative analysis of crystal structures of mitochondrial cytochrome $b c_{1}$ with bound substrate and inhibitors at the $\mathrm{Q}_{i}$ site., Biochemistry 42 (2003) 9067-80. doi:10.1021/bi0341814.

[18] C. Lange, J.H. Nett, B.L. Trumpower, C. Hunte, Specific roles of protein-phospholipid interactions in the yeast cytochrome $b c_{1}$ complex structure, EMBO J. 20 (2001) 65916600. doi:10.1093/emboj/20.23.6591.

[19] C. Hunte, J. Koepke, C. Lange, T. Roßmanith, H. Michel, Structure at $2.3 \AA$ resolution of the cytochrome $b c_{1}$ complex from the yeast Saccharomyces cerevisiae cocrystallized with an antibody Fv fragment, Structure 8 (2000) 669-684.

doi:10.1016/S0969-2126(00)00152-0. 


\section{Legends to figures}

Fig.1 Location of the mutated residues in the $\mathrm{Q}_{\mathrm{i}}$ domain of the $b c_{1}$ complex. Cytochrome $b$ is shown as a ribbon and water molecules as black dots. UQ, Ubiquinone; CDL, Cardiolipin. The figure was drawn using the structure PDB : 1KB9. The residues mutated in PFQi11 and the suppressors are shown.

Fig.2 Growth competence of WT and mutants. Cell suspensions were spotted on YDP and YPG medium and incubated for two days (YPD) or three days (YPG).

Fig.3 Oxygen consumption rates of WT and mutant cells, and effect of uncoupler. Oxygen consumption rate were measured as described in Materials and Methods with and without the uncoupler CCCP. The measurements of WT, PFQi11 and the double mutant F225L-K228L (FL-KL) have been repeated seven times and with cells from three different cultures; the measurements with the other strains were repeated three times. Valued were averaged. The error bars represent standard deviations. A) Oxygen consumption rates $\left(\mathrm{mM} \mathrm{O}_{2}\right.$ per min per $\mathrm{OD}_{600 \mathrm{~nm}}$ cells) without $\mathrm{CCCP}$; B) Fold changes between oxygen consumption rates with and without CCCP.

Fig.4 Activity of the ROS-sensitive aconitase in WT and mutant cells. Aconitase and fumarase activities were assessed spectrophotometrically as described in Materials and Methods. The data are presented as the ratio of the aconitase activity and the fumarase activity. Values are the averages of four-five independent experiments, with error bars representing standard deviation. 
Table $1 b c_{1}$ complex activity in WT and mutants

\begin{tabular}{|l|l|l|l|}
\hline strain & \multicolumn{1}{|c|}{ mutations } & $\mathrm{k}_{\mathrm{cat}}\left(\mathrm{s}^{-1}\right)$ & $\mathrm{K}_{\mathrm{M}}(\mu \mathrm{M})$ \\
\hline WT & & $53+/-2$ & 3 \\
\hline F225L-K228L & F225L, K228L & $28+/-2$ & 7 \\
\hline PFQi11 & M221F, F225L, K228L,V233F & $48+/-2$ & 7 \\
\hline SUP1 & M221F, F225L, K228L, T232I, V233F & $50+/-6$ & 4 \\
\hline SUP2 & N31D, M221F, F225L, K228L,V233F & $27+/-3$ & 4 \\
\hline SUP3 & Y28S, M221F, F225L, K228L,V233F & $43+/-2$ & 4 \\
\hline SUP5 & M221F, F225L, K228M,V233F & $40+/-2$ & 5 \\
\hline SUP6 & M221F, F225, K228L,V233F & $55+/-4$ & 3 \\
\hline
\end{tabular}

$b c_{1}$ complex activities were determined by measuring the reduction of cytochrome $c$ using broken mitochondria. $k_{c a t}$ are cytochrome $c$ reduction rates per $b c_{1}$ complex. $\mathrm{K}_{\mathrm{M}}$ were estimated from the plots of cytochrome $c$ reduction rates $v s$ decylubiquinol concentrations, as the decylubiquinol concentrations required to obtain $50 \%$ of the observed maximum rate of cytochrome $c$ reduction 


\section{Figure 1}

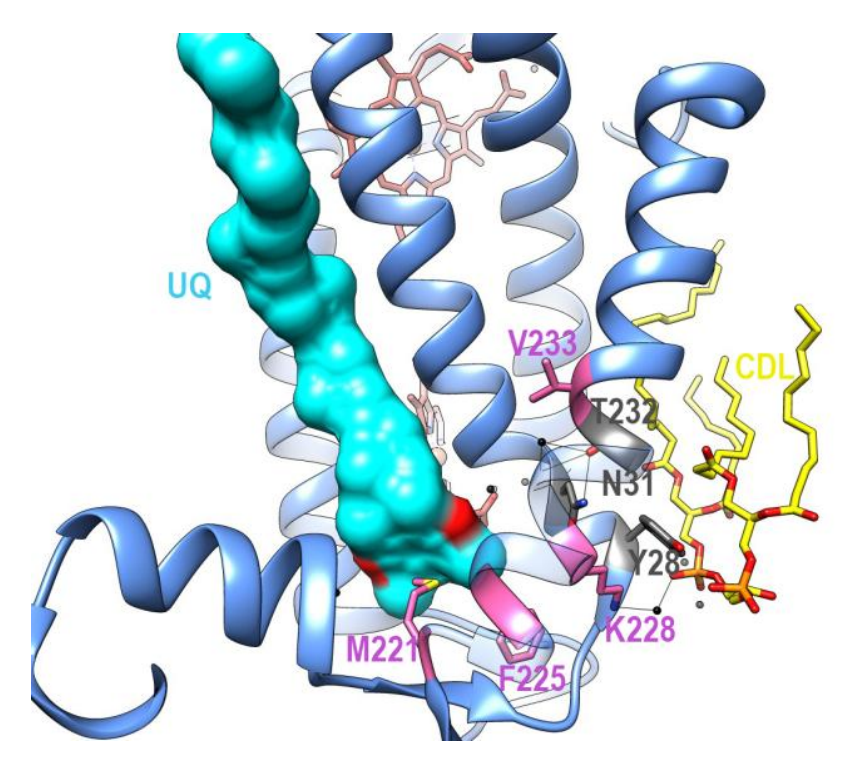


Figure 2

尊



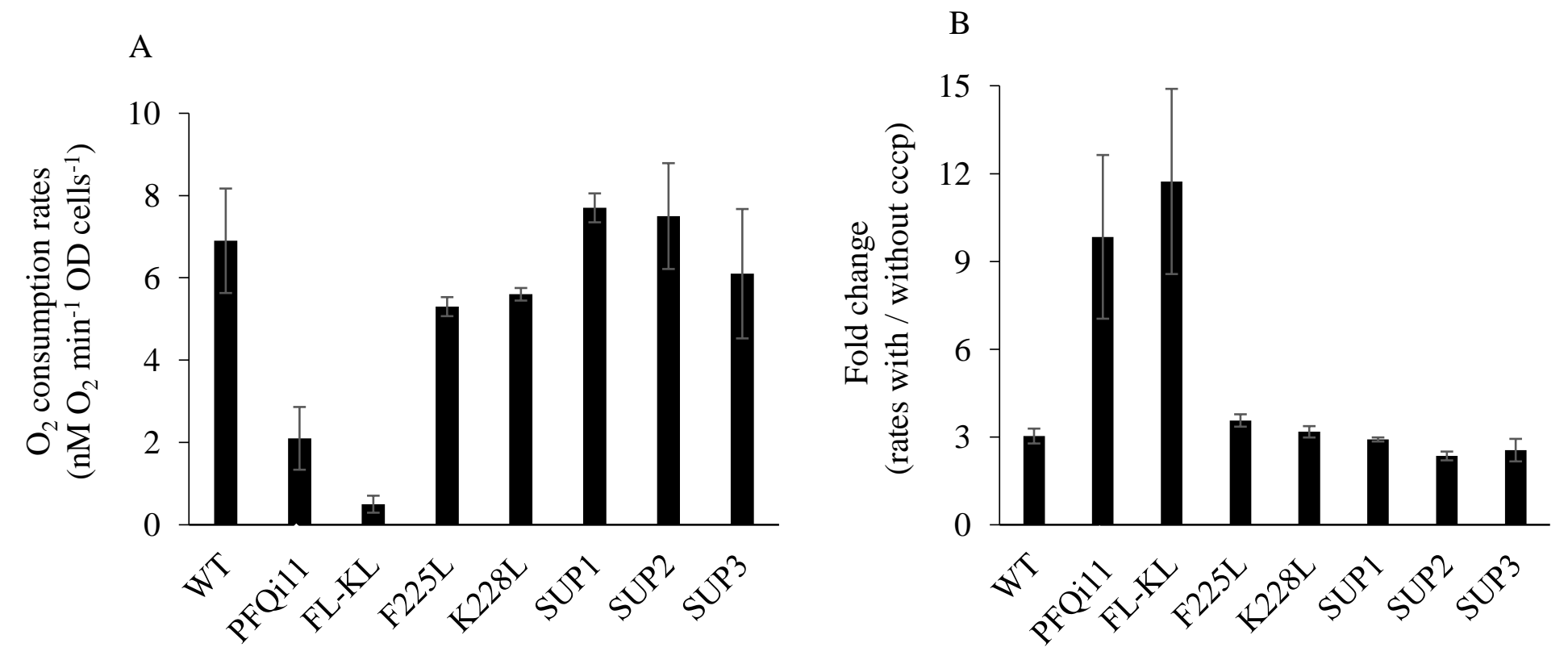
Figure 4

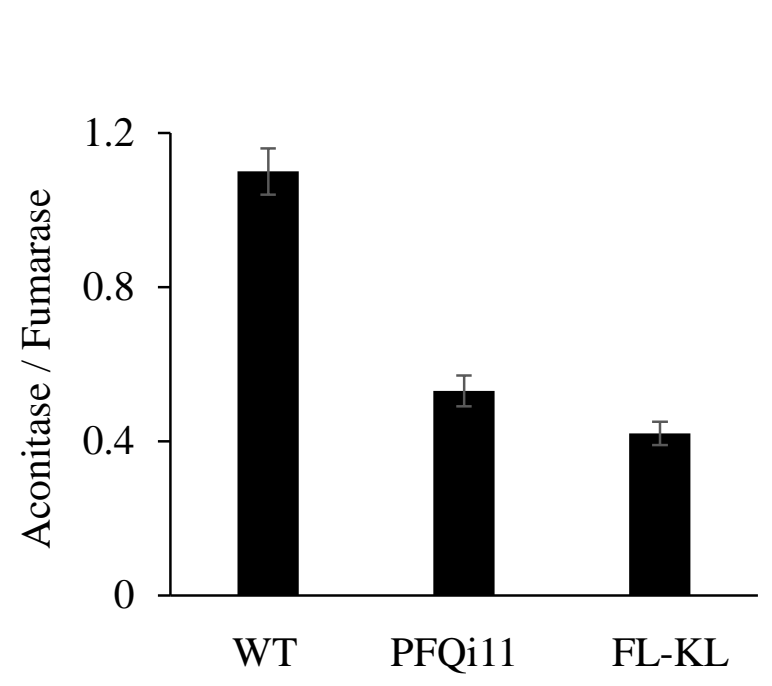

